\title{
The Activity Modalities: A Priori Categories of Coordination
}

\author{
Lars Taxén
}

\section{Linköping University Post Print}

\section{Tweet}

N.B.: When citing this work, cite the original article.

Original Publication:

Lars Taxén, The Activity Modalities: A Priori Categories of Coordination, 2015, Proceedings of the Fourth International Conference on Cognitive Neurodynamics - 2013, (1), 21-29. http://dx.doi.org/10.1007/978-94-017-9548-7_4

Copyright: Springer

Postprint available at: Linköping University Electronic Press

http://urn.kb.se/resolve?urn=urn:nbn:se:liu:diva-112852 


\title{
The Activity Modalities - A-priori Categories of Coordination
}

\author{
Lars Taxén
}

Linköping University

E-mail: lars.taxen@gmail.com

\begin{abstract}
A conceptualization of a-priori forms of coordination as activity modalities is proposed. Sensations in various sensory modalities are integrated by our brain into a coherent, actionable percept, structured by the processes of objectivation, contextualization, spatialization, temporalization, stabilization, and transition. This conceptualization promises to bridge neuroscientific and applied research into the coordination problem.
\end{abstract}

\section{Introduction}

The understanding of how actions are coordinated and integrated in the pursuit of fulfilling needs is of prime importance for brain research:

"I do not see any way to avoid the problem of coordination and still understand the physical basis of life" [1, p. 176]

Coordination has been extensively investigated "internally" in various neuroscientific and cognitive studies, and "externally" in applied areas like, for example, organizational studies. Usually, neuroscientific research focuses of the inner workings of the brain, while the external environment is conceptualized in rather general, non-specific terms. As a case in point, see Figure 1:

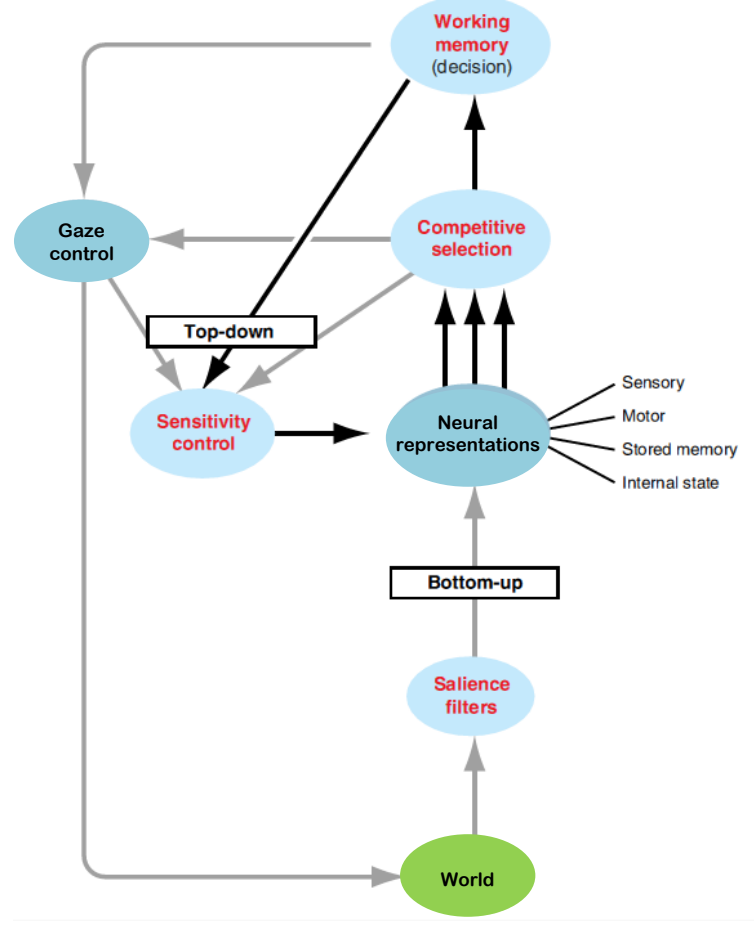

Figure 1: An example of conceptualizing the brain - environment relation (adapted from [2])

The brain is modeled in an elaborate way, while the environment is simply described as the "world". Thus, it is recognized that neural representations are influenced by the external world, but the character of these representations is not problematized. Also, influences in the opposite direction - from neural representations to the "world" - are not considered.

In a similar vein, "external" sciences such as organizational science, social sciences, information system development, system engineering, project management, etc., tend to regard the individual as a homogeneous ideal type that can be analyzed and manipulated as other, nonhuman elements. Thus, human abilities and limitations for acting are disregarded, which may have severe consequences for research in areas where humans are relevant. For example, models of organizations are often so complex that they are exceptionally hard to overview, understand and agree upon (see e.g. TOGAF [3]; a framework for developing so called enterprise architectures).

The separation between the internal and external research areas is understandable since research efforts have to be somehow delimited. However, this dichotomy needs to be overcome in order to advance our understanding of coordination. The brain, body and the environment should be seen in a unitary way, as succinctly stated by Llinás [4]:

"[The] internal functional space that is made up of neurons must represent the properties of the external world - it must somehow be homomorphic with it" [4, p. 65]

Concerning coordination, it is reasonable to assume that every healthy human being is born with certain phylogenetically evolved predispositions to coordinate actions in the same sense that we are born with legs for walking. Such predispositions need to be ontogenetically developed by acting in the various situations that the individual encounters during her life-span. These actions are manifested internally as a changed state of mind, and externally as various means enabling the actions. For example, the ability to orientate oneself in space is certainly alleviated by a map, once the significance of it has been understood by the individual.

The purpose of this contribution is to suggest that the homomorphy between the internal and external worlds can be conceptualized as activity modalities. These modalities - objectivation, contextualization, spatialization, temporalization, stabilization, and transition - should be conceived as a-priori forms, i.e. exigencies for coordinating actions in the same vein that Kant suggested time and space as a-priori forms for knowledge. Thus, the modalities are not something that can be experienced or sensed in the external world. Rather, they are evolutionary developed categories by which our brains are equipped for enabling action. The gist of this position is that sensations in various sensory modalities are integrated by our brain into a coherent, actionable percept structured by the activity modalities and their interdependencies. 
The activity modality construct was conceived in my work with the coordination of extremely complex development projects in the telecommunication industry [5]. Gradually, after many years, external manifestations such as information models (spatialization), process models (temporalization), business rules (stabilization), as well as other organizational artifacts, were elaborated into the activity modality construct as putative, general categories of coordination.

The rest of the paper is organized as follows. First, I illustrate the activity modalities by the activity of ancient time mammoth hunting. Next, in order to provide a "foothold" from the external world into the intricacies of the inner world, I suggest modeling the brain as dependencies between capabilities. This approach is inspired from efficacious attempts to capture the essentials of complex systems in the telecom industry [6]. The paper is concluded with a discussion of the implications of the activity modality approach. In conclusion, I suggest that this line of thinking is promising enough for motivating further research efforts along this path.

\section{The activity modalities}

Imagine that you can travel some 30000 years back in time, and you are one of the hunters in Figure 2, motivated by the need to get food, acquire material for clothing, making arrowheads, and the like. What coordinative capabilities must you have in order to participate in this activity ${ }^{1}$

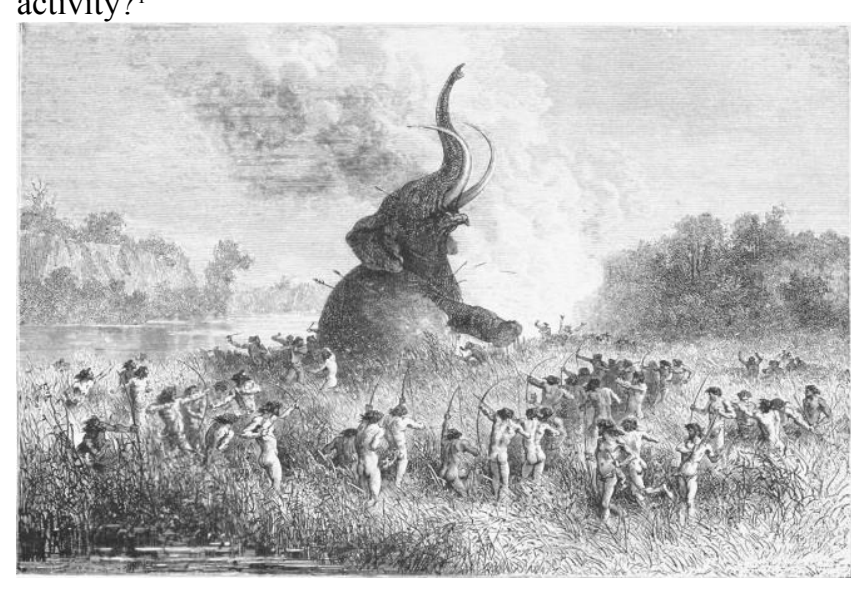

Figure 2: Illustration of an activity (Original wood engraving by E Bayard [8]).

A basic ability is that you can direct your attention to the object in focus for the activity - the mammoth. If you are unable to recognize the very target that your actions are meant to affect, you cannot coordinate your actions with the other hunters.

Second, given the object and the motive for acting, you need to conceive of a context - a center of gravity so to say - that enables you to recognize that which is relevant to the activity (and disregarding irrelevant things): hunters, bows, arrows, actions, shouts, gestures, and so on. For example, the river in the background is certainly

1 “Activity" (German: Tätigkeit; Russian: deyatel'nost') as used here refers to the rather specific sense it has in Activity Theory [7], meaning roughly "socially organized work". Thus, it is more precise than every-day English understanding of "activity". By "action" I refer to the actions of individuals participating in an activity. relevant, since it is obstructs the mammoth from escaping in that direction. On the other hand, from participating in fishing activities you know that there are fishes in the river; but these are surely irrelevant in the mammoth hunting activity.

The context is fundamental for making sense of individual actions. For example, it can be seen in the background of Figure 2 that beaters scare the pray away by means of making noises and a lit fire. These actions would appear completely counterproductive if seen in isolation. It is only in the context of the entire activity that the beaters' actions become intelligible.

Third, a sense of the spatial structure of the activity needs to be developed in your mind. This enables you to recognize how the relevant things are related to each other, and what properties you confer upon them.

Fourth, you must acquire a sense for the temporal or dynamic structure of the activity. Your actions must be carried out in a certain order. For example, shooting an arrow involves the steps of grasping the arrow, placing it on the bow, stretching the bow, aiming at the target, and releasing the arrow.

Fifth, you cannot shoot your arrows in any way you like. If shooting in a wrong direction, other hunters may be hit rather than the mammoth. You need to know where to aim in order to hurt the mammoth the most. An understanding of how to perform appropriate mammoth hunting will be accrued after many successful (and, presumably, some less successful) mammoth hunts. Eventually, this lends a sense of stability to the activity; of the "taking for granted"; rules and norms indicating proper patterns of action that need not be questioned as long as they work.

Sixth, an activity is typically related to other activities. For example, the prey will most likely be cut into pieces and prepared to eat. This is done in a cooking activity, which in turn has its particular motive - to still hunger and object, which happens to be the same as for the hunting activity: the mammoth. However, in this context, other aspects of the mammoth are relevant (as, for example what parts of the mammoth are edible). In order to participate in or conceive of other activities, you must be capable of refocusing your attention; to make a transition from one activity to another.

The six dimensions outlined above - objectivation, contextualization, spatialization, temporalization, stabilization, and transition between contexts - are denoted activity modalities. In order for an individual to coordinate her actions, her brain must be able to integrate multimodal sensory impressions into an actionable Gestalt in the form of the activity modalities and their interdependencies. This integration may be precluded by neurological deficiencies. For example, a brain lesion in the hippocampal area severely impairs spatial navigation, which in turn impedes orientation towards a desired target [9].

Even if coordinative capabilities are strictly individual, our social and physical environment is imperative for how these capabilities are manifested in the brain. Coordination between individuals can only occur through external means that enable individual actions to be sufficiently synchronized. Such means can be anything relevant for the activity: tools, speech, writings, gestures, symbols, and whatever else as long as they are pertinent 
for the coordination and integration of actions in order to achieve a common goal. This indicates that the activity should be the prime unit of analysis for coordinative inquiries. In order to pursue this line of thinking, I have suggested denoting activities characterized by the activity modalities as activity domains [5].

The idea behind illustrating the activity modalities by a historical activity such as a mammoth hunt is to convey that the underlying structure of activity domains is the same, regardless of time and place. The reason is simply that our phylogenetic constitution has not changed much (if at all) since we were hunting mammoths in ancient times. However, contemporary ontogenetic manifestations of the modalities are of course very different, not least because of the technological development of means.

\section{An "anatomy" of the brain}

If we hypothesize that the activity modalities are relevant for inquiring about coordination, we need to investigate how these capabilities are realized in the brain. In order to address this task, I propose to model the brain as dependencies between capabilities; from the most basic ones upwards.

This approach is inspired by experiences from managing the coordination of extremely complex development tasks in the telecom industry, where the system under development was conceived in precisely this way [6]. The principle behind is quite simple: the most important thing for understanding how a complex system works is to understand dependencies. Images illustrating such dependencies have, perhaps somewhat impertinent, been coined "anatomies" since it captures how the system "comes alive". So, for example, if the power on button of a computer is malfunctioning, none of the inherent capabilities of the computer can be actuated.

In Figure 3, such an "anatomy" of the brain is shown:

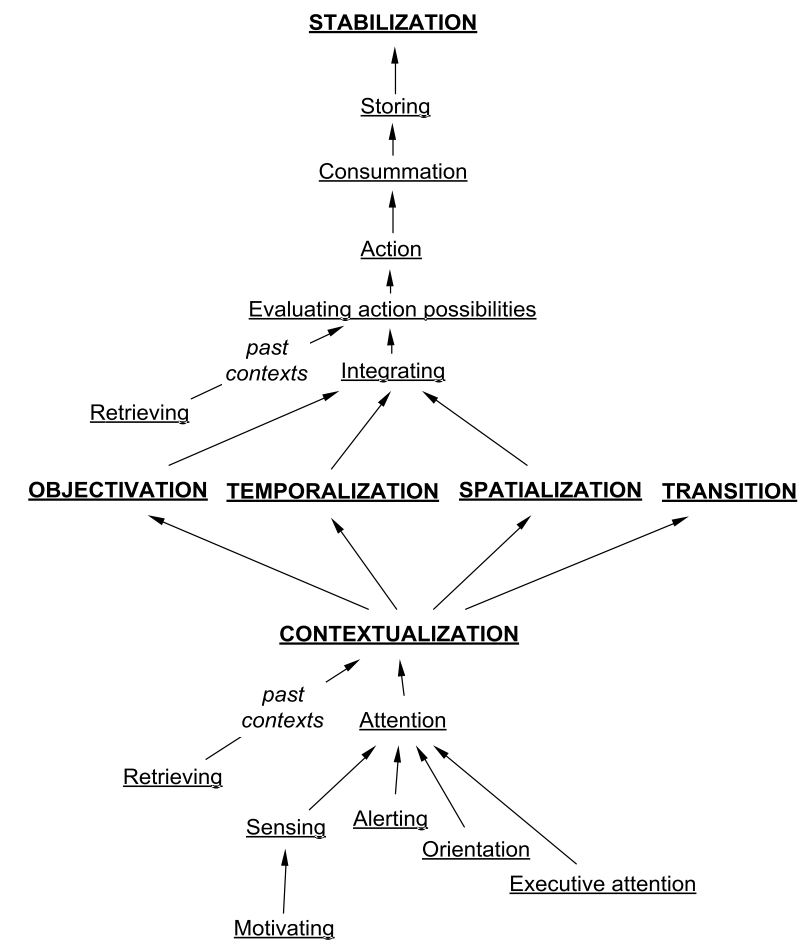

Figure 3: Dependencies between capabilities in the brain.

This anatomy should be regarded as a highly speculative and preliminary first attempt that needs further elaboration. Its character is strictly structural; there is no aspect of time or dynamics involved. Also, it is meant to illustrate the phylogenetic predispositions which are further elaborated into coordinative capabilities during ontogenesis. Thus, the ensuing capabilities of the individual are realized by the interplay between these predispositions and external influences from the environment.

How the capabilities are realized in Figure 3 is not illustrated. This may appear strange at first glance, since the anatomy is meant to assist in finding out exactly that. However, the purpose of the anatomy is to provide a model of the whole of the brain; a model that is simple enough, yet adequate, for achieving a common understanding about it.

The anatomy should be read from the bottom up. A basic brain capability is the motivating one, indicating that the brain is capable of auto-activation and continual exploration of the environment. Next, a sensing capability is necessary, which is realized by the different sensory systems in the brain (visual, auditory, somatosensory, gustatory, and olfactory ones).

Sensing is a prerequisite for attention, which in turn depends of the capabilities alerting (achieving and maintaining a state of high sensitivity to incoming stimuli), orientation (the selection of information from sensory input), and executive attention (monitoring and resolving conflict among thoughts, feelings, and responses) [10].

The contextualization capability is dependent on attention and the capability to resolve ambiguous percepts, which requires retrieval of akin percepts from long-term memory: "analogies are derived from elementary information that is extracted rapidly from the input, to link that input with the representations that exist in memory." [11, p. 1235].

With contextualization in place, the objectivation, spatialization, and temporalization capabilities can be invoked. In particular, it can be noted that objectivation depends on contextualization; indicating that the discrimination of an object in focus requires a contextual background. The transition modality is modeled as being dependent on contextualization, since transition is not possible if the contextualization capability is inhibited.

Next, the integration capability can be actuated, i.e., the formation of a pre-motor, actionable percept, which enables the evaluation of proper action alternatives utilizing similar percepts retrieved from long term memory. After the motor system executes the action, its consequences are evaluated by a "consummation" capability, and the experience is stored in long term memory. This in turn is a prerequisite for the stabilization capability.

\section{Discussion and conclusions}

The approach presented in this paper is an attempt to address "The most important issue in brain research

\footnotetext{
2 Consummation is the last stage in Mead's conceptualization of the act "upon which the encountered difficulty is resolved and the continuity of organic existence re- established" [12, p. 3-25]
} 
today $[\ldots$,$] that of the internalization or embedding of$ the universals of the external world into an internal functional space" [4, p. 64].

The basic idea put forward is that our individual capabilities for action will inevitably reflect how we construct our artifacts and social institutions. Thus, the "universals of the external world" are not something that exists out there before mankind appeared on earth; rather these reflect "universals" of our brains, which evolution has brought about. This means that the activity modalities can be apprehended as "coordinative universals" by which our internal and external worlds are unified.

In fact, the capabilities that the activity modalities provide are so vital for our lives that they remain unnoticed by us, like the air we breathe. Every moment of our daily life, we effectuate these capabilities without reflection. It is only when we are struck by some lesion that the lack of a modality capability becomes apparent.

There exists a substantial amount of research that substantiates the idea of the activity modalities in the "external world" e.g. [5; 6]. However, there is a huge gap in extant research regarding how the modalities are realized in our brains. In spite of this, some interesting threads can be noticed. For example, the stabilization modality implies that every activity needs to uphold a delicate balance between the extremes of complete segregation and all-encompassing integration. At one extreme, the activity is disintegrated into non-communicating fragments, and at the other extreme, the activity is solidified and unable to change. This view has a striking resemblance to the concept of metastability:

"Metastability, [...] leads to a looser, more secure, more flexible form of function that can promote the creation of new information. [...]Too much autonomy of the component parts means no chance of coordinating them together. On the other hand, too much interdependence and the system gets stuck, global flexibility is lost." [13, p. 43]

Another putative connection is the formation of "global neurocognitive state" which "plays a critical role in adaptive behavior by allowing the organism to perceive and act in a manner consistent with the context of the changing situation in which it exists" [14, p. 61]. An idea worth pursuing is that the dynamical assessment of the state of an organism can be conceptualized, at least concerning coordination of actions, as activity modalities.

Concerning the grounding of the activity modality construct, relevant results from the immense knowledge base that exist in neuroscientific research today need to be addressed. In order to coordinate these results, a brain anatomy such as the one in Figure 3, might be used. Such an image would function as a common target where pertinent research results can be related to different capabilities in the anatomy. After all, such images have been successfully used in coordinating other complex tasks!

I suggest that the activity modality approach may provide insights both in the internal and external worlds. In the external world, we may use the activity modalities as a guiding framework for analyzing and constructing our social and physical environment. Likewise, we may study the nature of activity modality manifestations such as artifacts, norms, institutions, etc., in order to gain insight into the coordinative, functional organization of the brain. In conclusion, I claim that the conceptualization of coordination proposed in this contribution is promising enough to motivate further research efforts along this path.

\section{References}

[1] Pattee, H.H. (1976). Physical theories of biological coordination. In M. Grene \& E. Mendelsohn (Eds.), Topics in the philosophy of biology, 27, Boston: Reidel.

[2] Knudsen, E.I. (2007). Fundamental Components of Attention. Annual Review of Neuroscience, 30, 5778.

[3] TOGAF (2013). Retrieved July $28^{\text {th }}$, 2013, from http://pubs.opengroup.org/architecture/togaf9doc/arch/

[4] Llinás, R.R. (2001). I of the vortex: from neurons to self. Cambridge, Mass.: MIT Press.

[5] Taxén, L. (2009). Using Activity Domain Theory for Managing Complex Systems. Information Science Reference. Hershey PA: Information Science Reference (IGI Global).

[6] Taxén, L. (Ed.) (2011). The System Anatomy - Enabling Agile Project Management. Lund: Studentlitteratur. ISBN 9789144070742.

[7] Kaptelinin, V., \& Nardi, B. (2006). Acting with Technology - Activity Theory and Interaction Design. Cambridge, MA: The MIT Press.

[8] Bryant, W. C., Gay, S. H., (1983). A Popular History of the United States. Vol. I, Charles Scribner's Sons, New York.

[9] Posner, M.I., \& Petersen, S.E. (1990). The Attention System of the Human Brain. Annual Reviews of Neuroscience, 13, 25-42.

[10] Posner, M.I., and Rothbart, M.K. (2007). Research on Attention Networks as a Model for the Integration of Psychological Science. Annual Review of Psychology, 2007 (58), 1-23.

[11] Bar, M. (2009). The Proactive Brain: Memory for Predictions. The Philosophical Transactions of the Royal Society, (364), 1235-1243.

[12] Mead, G.H. (1938). Philosophy of the act. Chicago: University of Chicago Press.

[13] Kelso, J. A. S, \& Tognoli, E. (2007). Toward a Complementary Neuroscience: Metastable Coordination Dynamics of the Brain. In L. I. Perlovsky, R. Kozma(Eds.) Neurodynamics of Cognition and Consciousness (pp. 39-59). Berlin Heidelberg: Springer.

[14] Bressler, S.L. (2007). The Formation of Global Neurocognitive State. In L. I. Perlovsky, R. Kozma(Eds.) Neurodynamics of Cognition and Consciousness (pp. 61-72). Berlin Heidelberg: Springer. 